\title{
Continuation of Biharmonic Functions across Circular Arcs*
}

\author{
JAMES H. BRAMBLE
}

\author{
Communicated by C. Loewner
}

\begin{abstract} ing conditions are satisfied:
(A)

$$
\begin{aligned}
w=M(w)=0 & \text { on } Q, \\
M(w)=V(w)=0 & \text { on } Q, \\
w_{r}=V(w)=0 & \text { on } Q,
\end{aligned}
$$
\end{abstract}

This paper establishes certain reflection principles (analogous to the classical Schwarz reflection principle for harmonic functions) for biharmonic functions subject to various boundary conditions. Explicit formulæ are given for the continuation of a biharmonic function $w$ across a circular are $Q$ when the follow-

where

$$
M(w)=\Delta w+\frac{1-\sigma}{\sigma} w_{r r}
$$

and

$$
V(w)=\frac{\partial}{\partial r}(\Delta w)+(1-\sigma) \frac{1}{a^{2}}\left[w_{\theta \theta r}-\frac{1}{a} w_{\theta \theta}\right],
$$

$\Delta$ being the Laplace operator and $a$ the radius of the circle. If $\sigma$ is taken to be Poisson's ratio, then (A) and (B) correspond physically to the cases where $Q$ is the edge of an elastic plate which is "simply supported" and "free" respectively. Condition (C) corresponds to the so-called "sliding clamped" case.

*This research was supported by the United States Air Force through the Air Force Office of Scientific Research of the Air Research and Development Command under Contract No. AF18(600)-573. 


\section{InTRODUCTION}

The classical Schwarz reflection principle [30] may be stated as follows. Let $R$ be an open subset of $x>0$, a portion $L$ of whose boundary is a non-empty, simply connected, open set in $x=0$. Define $R^{*}$ to be the set of all points $p(x, y, z)$ such that either $p(x, y, z) \varepsilon R$ or $p(0, y, z) \varepsilon L$. If $u$ is harmonic in $R$ and assumes the boundary value zero on $L$ (that is $\lim _{(x, y, z) \rightarrow\left(0, y^{\prime}, z^{\prime}\right)} u(x, y, z)=0$, where $(x, y, z) \varepsilon R$ and $\left.\left(0, y^{\prime}, z^{\prime}\right) \varepsilon L\right)$ then $u$ may be continued so as to be harmonic in $R^{*}$ by the relation

$$
u(-x, y, z)=-u(x, y, z) .
$$

Clearly this statement is valid also in two dimensions.

Several analogous reflection principles for plane boundaries have been found. For example, it is well known that if $\partial u / \partial x$ assumes the boundary value zero on $L$ then (1.1) may be replaced by

$$
u(-x, y, z)=u(x, y, z) .
$$

Also, formulæ for the continuation of harmonic functions across spherical surfaces (circular ares) are known. These formulæ for reflection depend, of course, on the given boundary conditions (see Section II, Lemmas 1 and 2).

Hoheisel [15] and later Poritsky [26] consider the boundary condition $\partial u / \partial x-a u=0$ on $L$ where $a$ is a constant, and from this derive the continuation formula for the harmonic function $u$. Analogous reflection principles for linear elliptic second order partial differential equations with constant coefficients have been deduced by Diaz \& LUDFord [7].

A reflection principle was given by PoRITsKy [28] for biharmonic functions in the plane satisfying $u=0, \partial u / \partial n=0$ (clamped edge-see [33, p. 235]) along a straight line. This problem was considered later by DuFFIN in [8] where he states precisely the conditions under which such a continuation is possible. In [8] he also considers the "simply supported" and "free" (see [33, p. 235]) boundary conditions for the case of the plane boundary and the "clamped" condition for the spherical boundary. In each instance an explicit formula is given.

DuFris also obtains formulæ for the continuation of slow steady viscous flows, and solutions of the equations of elasticity subject to both the "free" and "fixed" (see [9]) boundary condition. Only the plane boundary is treated. Analogous continuation formulæ have been obtained by BRAMBLE (Continuation of Solutions of the Equations of Elasticity, in print).

HUBER [16] gives a reflection principle for equations of high order. He derives a formula for the continuation of a polyharmonic function (of order $p$ ) across a plane boundary (portion of $x=0$ ) provided

$$
\lim _{P \rightarrow Q} \frac{w(P)}{x^{p-1}}=0
$$

where $Q$ is any point on $x=0$. In [17] he gives a reflection principle for solutions 
of

$$
\left(\frac{\partial^{2} u}{\partial x_{1}^{2}}+\frac{\partial^{2} u}{\partial x_{2}^{2}}+\cdots+\frac{\partial^{2} u}{\partial x_{n}^{2}}\right)+\frac{K}{x_{n}} \frac{\partial u}{\partial x_{n}}=0
$$

( $K \geqq 1$ ) across the hyperplane $x_{n}=0$. For discussions of a somewhat more general nature see JoHN [18] and Garabedian [13].

In the present paper continuation formulæ analogous to those given by DUFFIN [8] for the plane boundary are proved for the circular boundary in the biharmonic case. In addition, the "sliding clamped" condition is also considered.

\section{Definitions and Lemmas}

The following notation will be employed. The symbol $\Delta$ will denote the two dimensional Laplace operator, i.e.

$$
\Delta=\frac{\partial^{2}}{\partial x_{1}^{2}}+\frac{\partial^{2}}{\partial x_{2}^{2}}
$$

This operator, written in polar coordinates, is

$$
\Delta=\frac{1}{r} \frac{\partial}{\partial r}\left(r \frac{\partial}{\partial r}\right)+\frac{1}{r^{2}} \frac{\partial^{2}}{\partial \theta^{2}}
$$

A function $h$, satisfying $\Delta h=0$ in a given region $R$, is said to be harmonic provided $h_{\varepsilon} C^{2}$ throughout $R$. A function $b$, satisfying $\Delta^{2} b \equiv \Delta(\Delta b)=0$ in $R$, is said to be biharmonic provided $b \varepsilon C^{4}$ in $R$. All functions occurring in this paper termed harmonic or biharmonic are assumed to be single valued.

If one of the coordinates occurs as a subscript it denotes partial differentiation with respect to this subscript, e.g. $h_{r} \equiv \partial h / \partial r$.

In the usual manner, $r$ will represent the distance from a point to the origin of the coordinate system, i.e. $r=\sqrt{x_{1}^{2}+x_{2}^{2}}$. We define $\bar{x}_{i}=a^{2} x_{i} / r^{2}$ and $R=$ $\sqrt{\bar{x}_{1}^{2}+\bar{x}_{2}^{2}}$ where $a$ is a positive constant. (Note that $R r=a^{2}$.)

Often $p$ will be used to denote the point having coordinates $(r, \theta)$ and $p^{\prime}$ for the point with coordinates $(R, \theta)$. The two points $p$ and $p^{\prime}$ are said to be inverse points relative to the circle $r=a$.

We define $D^{*}$ to be a two dimensional connected region which excludes the origin but which contains a portion $Q$ of a circle of radius $a$ with center at the origin, and such that if a point with coordinates $(r, \theta)$ is in $D^{*}$ then the point $(R, \theta)$ is also in $D^{*}$. Furthermore, $D^{*}$ is to have the property that every ray from the origin which intersects $D^{*}$ intersects it in a single line segment. The portion of $D^{*}$ which belongs to the open disc $r<a$ will be called $D$. It is necessary for the present to require $D$ to be simply connected. However, it will be shown later that the continuation formulæ remain valid when this restriction is removed.

Let $h$ be harmonic (biharmonic) in $D$. A function $h^{*}$ is said to be a harmonic (biharmonic) continuation of $h$ into $D^{*}$ provided $h^{*} \equiv h$ in $D$ and $h^{*}$ is harmonic 
(biharmonic) in $D^{*}$. If such a function $h^{*}$ can be found then $h$ is said to have been continued harmonically (biharmonically) into $D^{*}$.

In this paper whenever we write $f(r, \theta)=0$ on $Q$, we mean $f(r, \theta)$ assumes the boundary value zero on $Q$, i.e. $\lim _{(r, \theta) \rightarrow\left(a, \theta^{\prime}\right)} f(r, \theta)=0$ where $(r, \theta) \varepsilon D$ and $\left(a, \theta^{\prime}\right) \varepsilon Q$.

The following lemmas will be needed.

Lemma 1: Let $\phi$ be harmonic in $D$ and satisfy $\phi=0$ on $Q$. Then $\phi$ may be continued into $D^{*}$ by the relation

$$
\phi(R, \theta)=-\phi(r, \theta)
$$

This is the classical reflection principle.

Lemma 2: Let $\phi$ be harmonic in $D$ and satisfy $\partial \phi / \partial r=0$ on $Q$. Then $\phi$ may be continued into $D^{*}$ by the relation

$$
\phi(R, \theta)=\phi(r, \theta) .
$$

This follows from Lemma 1 and the fact that if $\phi$ is harmonic in $D, r \partial \phi / \partial r$ is also harmonic in $D$.

Remark. Because of the analytic character of harmonic functions, the continuations given in Lemmas 1 and 2 are necessarily unique [19, p. 259]. Thus we may state the following lemma.

Lemma 3: Let $\phi$ be harmonic in $D$ and satisfy $\phi=0, \partial \phi / \partial r=0$ on $Q$. Then $\phi \equiv 0$ in $D$.

After a careful search the author was unable to find the following two lemmas in the literature. These lemmas are of considerable importance in the development of the later theorems.

Lemma 4: Let $\phi$ be harmonic in $D^{*}$. Then there exists a harmonic function $\psi$ such that

$$
k \psi+r \psi_{r}=\phi \text { in } D^{*}
$$

where $k$ is an arbitrary constant.

Proof. Define

$$
\psi^{*}(r, \theta)=-r^{-k} \int_{r}^{a} \rho^{k-1} \phi(\rho, \theta) d \rho .
$$

Then $k \psi^{*}+r \psi_{r}^{*}=\phi$ and

$$
\Delta \psi^{*}=\frac{a^{k}}{r^{k+2}}\left[a \phi_{r}(a, \theta)-k \phi(a, \theta)\right]
$$

in $D^{*}$.

Now define

$$
\psi=\psi^{*}-r^{-k} f(\theta)
$$


with $f(\theta)$ given by

$$
f(\theta)=\frac{a^{k}}{k} \int_{\theta_{0}}^{\theta} \sin k(\theta-\tau)\left[a \phi_{r}(a, \tau)-k \phi(a, \tau)\right] d \tau,
$$

where $\left(a, \theta_{0}\right)$ is a point on $Q$ and the integration is taken over $Q$. From the definition of $f$ and (2.4) it follows that $\Delta \psi^{*}=\Delta\left(r^{-k} f\right)$ in $D^{*}$ and hence from (2.5) we see that $\Delta \psi=0$ in $D^{*}$. It is clear that $\psi$ so defined satisfies (2.3).

In case $k=0$ the right hand side of (2.6) is to be considered in the sense of the limit. Under these conditions (2.6) becomes

$$
f(\theta)=\int_{\theta_{0}}^{\theta}(\theta-\tau) a \phi_{r}(a, \tau) d \tau .
$$

Remark: The requirement that $D$ be simply connected is important in the preceding proof. This can be seen from the following example. Let $D$ be the annular region $0<b<r<a$. Consider the case $k=1$ and take $\phi(r, \theta)=$ $\sin \theta / r$ in $D^{*}$. Then

$$
\psi^{*}=\frac{\sin \theta}{r} \ln \frac{r}{a},
$$

as defined, is a single valued function in $D$. Now from (2.6)

$$
f(\theta)=-2 \int_{0}^{\theta} \sin (\theta-\tau) \sin \tau d \tau
$$

where $\theta_{0}$ in this example may be chosen equal to zero. Clearly $f(\theta)$ is not single valued in $D$. Hence $\psi=\psi^{*}-f(\theta) / r$ is not a single valued function in $D$, although formally it satisfied the requirements of the theorem.

Lemma 5: Let $\phi$ be biharmonic in $D^{*}$. Then there exists a biharmonic function $\psi$ such that

$$
k \psi+r \psi_{r}=\phi \text { in } D^{*}
$$

where $k$ is an arbitrary constant.

Proof: Define

$$
\psi^{*}=-r^{-k} \int_{r}^{a} \rho^{k-1} \phi(\rho, \theta) d \rho .
$$

Then $k \psi^{*}+r \psi_{r}^{*}=\phi$ in $D^{*}$.

Now define $\psi$ as $\psi=\psi^{*}-r^{-k} f(\theta)$ with

$$
\begin{aligned}
f(\theta) & =\frac{a^{k}}{k} \int_{\theta_{0}}^{\theta} \sin k(\theta-\tau)\left\{\left[a \phi_{r}(a, \tau)-k \phi(a, \tau)\right]\right. \\
+ & \left.\frac{a^{2}}{k+2} \int_{\theta_{0}}^{\tau} \sin (k+2)(\tau-\sigma)\left[a(\Delta \phi)_{r}(a, \sigma)-(k+2) \Delta \phi(a, \sigma)\right] d \sigma\right\} d \tau
\end{aligned}
$$


where $\left(a, \theta_{0}\right)$ is a point on $Q$ and the integration is taken over $Q$. By direct computation it is easily seen that $\psi$ is biharmonic in $D^{*}$. Since

$$
k r^{-k} f(\theta)+r \frac{\partial}{\partial r}\left(r^{-k} f(\theta)\right)=0
$$

we have $k \psi+r \psi_{r}=\phi$ in $D^{*}$.

In case $k=0$ or $-2,(2.8)$ is taken in the sense of the limit and the proof is valid. Q.E.D.

(It was pointed out to the author by Professor C. Loewnen that Lemma 4 can be proved in a somewhat simpler manner by introducing complex variables.

Let $\varphi=\operatorname{Re} f(z)$ where $f(z)$ is analytic in $D^{*}$. The differential equation

$$
k g(z)+z g^{\prime}(z)=f(z)
$$

can be solved immediately for the analytic function $g(z)$. Thus, taking $\psi=$ $\operatorname{Re} g(z)$, the lemma follows.

Professor Loewner also pointed out that Lemma 5 may be proved by using Lemma 6 to write $\varphi=\alpha+r^{2} \beta$ with $\alpha$ and $\beta$ harmonic in $D^{*}$.

Now by Lemma 4 we can find harmonic functions $\lambda$ and $\mu$ such that $k \lambda+$ $r \lambda_{r}=\alpha$ and $(k+2) \mu+r \mu_{r}=\beta$. Lemma 5 follows by setting $\psi=\lambda+r^{2} \mu$.)

From the method of proof it is obvious that this lemma is true if $\phi$ and $\psi$ are polyharmonic of order $p$.

Corollary: Let $\phi$ be polyharmonic of order $p$ in $D^{*}$. Then there exists a polyharmonic function $\psi$ of order $p$ such that

$$
\phi=\sum_{i=0}^{n} a_{i} r^{i} \frac{\partial^{i} \psi}{\partial r^{i}}
$$

where the $a_{0}, \cdots, a_{n-1}$ are arbitrarily chosen constants and $a_{n}=1$. (Here $\partial^{0} \psi / \partial r^{0} \equiv \psi$.)

Lemma 6: Let $u$ be biharmonic in a simply connected region $R$. Then there exist harmonic functions $h_{1}$ and $h_{2}$ such that

$$
u=h_{1}+\left(r^{2}-a^{2}\right) h_{2} \text { in } R .
$$

This result is due to Boggro [2].

Other studies of the Almansi decomposition [1] were made for $n$ dimensions by Nicolesco [22, 23, 24], Colucci [4, 5], Picone [25], Fichera [10, 11, 12] and TolotTi [32].

Corollary. Let $u$ be biharmonic in $R$. Then there exist harmonic functions $h$ and $g$ such that

$$
u=h+\left(r^{2}-a^{2}\right)\left(g+k r h_{r}\right)
$$

in $R$ where $k$ is an arbitrarily chosen constant. 
Lemma 7: Let u be biharmonic in a simply connected, open subset $R$ of $D^{*}$ which contains $D+Q$ and be represented as

$$
u=h+\left(r^{2}-a^{2}\right)\left(g+k r h_{r}\right) \text { in } R
$$

with $\Delta h=0$ and $\Delta g=0$ in $R$. Then

$$
h=u-\left(r^{2}-a^{2}\right)\left[\frac{a}{r} V(a, \theta)-\frac{1}{4 r} \int_{r}^{a} \Delta u d \rho\right]
$$

and

$$
\begin{aligned}
g=\frac{a}{r} V(a, \theta) & -\frac{1}{4 r} \int_{r}^{a} \Delta u d \rho \\
& -k\left[r u_{r}-r \frac{\partial}{\partial r}\left\{\left(r^{2}-a^{2}\right)\left[\frac{a}{r} V(a, \theta)-\frac{1}{4 r} \int_{r}^{a} \Delta u d \rho\right]\right\}\right],
\end{aligned}
$$

where $V=g+k r h_{r}$.

Proof: From (2.12) and the definition of $V$ we have

$$
\frac{1}{4} \Delta u=V+r V_{r}=\frac{\partial}{\partial r}(r V) \text {. }
$$

Integrating (2.15) with respect to $r$ from $r$ to $a$ we have

$$
V=\frac{a}{r} V(a, \theta)-\frac{1}{4 r} \int_{r}^{a} \Delta u d \rho .
$$

Hence, solving (2.12) for $h$ and using (2.16), (2.13) follows. Substitution of (2.13) and (2.16) into the expression $g=V-k r h_{r}$ yields (2.14). Q.E.D.

Lemma 8: Let $\psi$ be a polyharmonic function of order $p$ in D. If $k \psi+r \psi_{r}$ $(k=$ constant $)$ can be continued into $D^{*}$ then $\psi$ can also be continued into $D^{*}$.

Proof: Define $u=k \psi+r \psi_{r}$. By hypothesis $u$ may be continued into $D^{*}$ and hence by the remark after Lemma 5 we may introduce a polyharmonic function $\psi^{*}$ (of order $p$ ) such that $k \psi^{*}+r \psi_{r}^{*}=u$ in $D^{*}$. Now in $D$ we have

$$
\frac{\partial}{\partial r}\left(r^{k} \psi\right)=\frac{\partial}{\partial r}\left(r^{k} \psi^{*}\right)
$$

Thus

$$
\psi=\psi^{*}+r^{-k} f(\theta)
$$

in $D$. But since the right hand side of (2.17) is defined and polyharmonic of order $p$ in $D^{*},(2.17)$ expresses a continuation of $\psi$ to $D^{*}$. Q.E.D.

Lemma 9: Let $h_{1}$ and $h_{2}$ be harmonic in $D$ and $w=h_{1}+\left(r^{2}-a^{2}\right) h_{2}$. If $w=0$ on $Q$ then $h_{1}=0$ on $Q$.

This lemma is similar to Lemma 3 [8, p. 316]. The proof is analogous. 
Lemma 10: Let $g$ be harmonic in $D$ and $u=c g+r g_{r}(c=$ constant $)$. If $u$ can be continued into $D$ then

$$
u\left(p^{\prime}\right)=c g\left(p^{\prime}\right)+R \frac{\partial g\left(p^{\prime}\right)}{\partial R}
$$

where $p \& D$.

Proof. By Lemma $8, g$ may be continued into $D^{*}$. Hence the continuation of $u$ into $D^{*}$ is expressed by (2.18). Q.E.D.

\section{Elastic Plate Problems}

We consider here the deflection $w$ of an elastic plate. We assume that $w$ is defined in a plane region $D$, a portion of whose boundary is circular. Expressions for the continuation of $w$ across $Q$ are obtained. The formulæ depend on the boundary values of $w$ on $Q$ and correspond to cases in which the plate is clamped, simply supported and free along $Q$. Also considered here is the so-called sliding clamped problem. The clamped problem for the circular boundary was first considered by DuFfin [8]. It is included here for the sake of completeness.

\section{Clamped Edge.}

Theorem 1: Let $w$ be defined in $D$ and satisfy

$$
\left.\begin{array}{rl}
\Delta^{2} w & =0 \text { in } D, \\
w & =0 \\
w_{r} & =0
\end{array}\right\} \text { on } Q .
$$

Then $w$ has a biharmonic continuation to $D^{*}$ given by

$$
w\left(p^{\prime}\right)=-w+r^{-2}\left(r^{2}-a^{2}\right)\left[r w_{r}-\frac{1}{4}\left(r^{2}-a^{2}\right) \Delta w\right] .
$$

\section{Simply Supported Edge.}

Theorem 2: Let $w$ be defined in $D$ and satisfy

$$
\left.\begin{array}{r}
\Delta^{2} w=0 \quad \text { in } \quad D, \\
w_{r r}+\frac{\sigma}{r} w_{r}=0 \\
w=0
\end{array}\right\} \text { on } Q .
$$

Then $w$ has a biharmonic continuation to $D^{*}$ given by

$$
\begin{aligned}
& w\left(p^{\prime}\right)=\left[(1-\sigma) \frac{\left(r^{2}-a^{2}\right)}{2 r^{2}}-1\right] w(p) \\
& +\frac{\sigma+1}{8}\left(r^{2}-a^{2}\right) r^{\frac{1}{2}(\sigma-3)} \int_{\tau}^{a}\left[2(\sigma-1) w(p)-\left(\rho^{2}-a^{2}\right) \Delta w(p)\right] \rho^{-\frac{1}{2}(\sigma+3)} d \rho .
\end{aligned}
$$


Proof: By Lemma 6, $w$ has the representation

$$
w=h+\left(\mathrm{r}^{2}-a^{2}\right) V
$$

in $D$, where $\Delta h=0$ and $\Delta V=0$ in $D$. Define $g$ as

$$
g=V+\frac{1}{2 a^{2}}\left(\frac{\sigma-1}{\sigma+1}\right) r h_{r} .
$$

Since $\Delta h=0, \Delta\left(r h_{r}\right)=0$ and hence $\Delta g=0$ in $D$. Substituting (3.8) into (3.7) we have

$$
w=h+\left(r^{2}-a^{2}\right)\left(g-\frac{1}{2 a^{2}}\left(\frac{\sigma-1}{\sigma+1}\right) r h_{r}\right) .
$$

Since $w=0$ on $Q$, by Lemma $9, h=0$ on $Q$ and, from Lemma $1, h$ is continued into $D^{*}$ by the relation

$$
h\left(p^{\prime}\right)=-h(p)
$$

Applying (3.4) to (3.9) and using Lemma 9 it follows that

$$
r g_{r}+\frac{1}{2}(1+\sigma) g=0 \text { on } Q \text {. }
$$

From Lemma $1, r g_{r}+\frac{1}{2}(1+\sigma) g$ can be continued into $D^{*}$ and hence, by Lemma $8, g$ can be continued to $D^{*}$. Since $h$ and $g$ can be continued as harmonic functions into $D^{*},(3.9)$ defines a biharmonic continuation for $w$ into $D^{*}$. For the remainder of the proof it is clear that we may assume that $w$ satisfies the boundary conditions in the strict sense.

To continue $g$ let

$$
u=r g_{r}+\frac{1}{2}(1+\sigma) g \text { in } D .
$$

Then $u$ satisfies $\Delta u=0$ in $D$ and $u=0$ on $Q$. By Lemma 1 such a function may be continued by defining $u\left(p^{\prime}\right)=-u(p)$. Substituting (3.12) into this expression, by Lemma 10 we have

$$
R \frac{\partial g\left(p^{\prime}\right)}{\partial R}+\frac{1+\sigma}{2} g\left(p^{\prime}\right)=-r \frac{\partial g(p)}{\partial r}-\frac{1+\sigma}{2} g(p) .
$$

We may rewrite this expression, using $R \partial / \partial R=-r \partial / \partial r$, to obtain

$$
\frac{\partial}{\partial r}\left[r^{-\frac{1}{2}(1+\sigma)}\left(g\left(p^{\prime}\right)-g(p)\right)\right]=(1+\sigma) r^{-\frac{1}{2}(3+\sigma)} g(p) .
$$

Integrating from $r$ to $a$ and noting that $g\left(p^{\prime}\right)=g(p)$ for $r=a$ we have

$$
g\left(p^{\prime}\right)=g(p)-(1+\sigma) r^{\frac{3}{2}(1+\sigma)} \int_{r}^{a} \rho^{-\frac{1}{2}(3+\sigma)} g(p) d \rho .
$$

Substituting (3.10), (3.14) into (3.9) and making use of $R \partial / \partial R=-r \partial / \partial r$ we arrive at the fact that 


$$
\begin{array}{r}
w\left(p^{\prime}\right)=-h(p)=\frac{a^{2}}{r^{2}}\left(r^{2}-a^{2}\right)\left[g(p)-(1+\sigma) r^{\frac{1}{2}(1+\sigma)} \int_{r}^{a} \rho^{-\frac{1}{2}(3+\sigma)} g(p) d \rho\right. \\
\left.-\frac{1}{2 a^{2}}\left(\frac{\sigma-1}{\sigma+1}\right) r h_{r}(p)\right]
\end{array}
$$

Expressions (2.13) and (2.14) with

$$
k=-\frac{1}{2 a^{2}}\left(\frac{\sigma-1}{\sigma+1}\right)
$$

may now be inserted into (3.15) and after a laborious but elementary computation (3.6) results. Q.E.D.

It is interesting to note here that although expressions (2.13) and (2.14) contain boundary terms the final expression (3.6) has none.

\section{Free Edge.}

Theorem 3: Let $w$ be defined in $D$ and satisfy

$$
\begin{gathered}
\Delta^{2} w=0 \text { in } D \\
\frac{\partial}{\partial r}(\Delta w)+(1-\sigma) \frac{1}{a^{2}}\left[w_{\theta \theta r}-\frac{1}{a} w_{\theta \theta}\right]=0
\end{gathered}
$$

and

$$
\Delta w+\frac{1-\sigma}{\sigma} w_{r r}=0 \text { on } Q .
$$

Then $w$ has a biharmonic continuation to $D^{*}$ given by

$$
\begin{aligned}
w\left(p^{\prime}\right)=\frac{1}{r} \int_{r}^{a} u\left(\frac{a^{2}}{\rho}, \theta\right) d \rho-\int_{r}^{a} \frac{1}{\rho} u\left(\frac{a^{2}}{\rho}, \theta\right) d \rho & \\
& -\frac{a}{r}(r-a) w_{r}(a, \theta)+w(a, \theta),
\end{aligned}
$$

where

$$
\begin{aligned}
u\left(\frac{a^{2}}{r}, \theta\right)= & \frac{\sigma-1}{\sigma+3}\left[\left(r^{2}+2 a^{2}\right) w_{r r}+\left(\frac{r^{2}-a^{2}}{r}\right)\left(r^{2} w_{r r}\right)_{r}\right] \\
& -\frac{\sigma-1}{4(\sigma+3)}\left(r^{2}-a^{2}\right)\left[\left(6-2 \frac{a^{2}}{r^{2}}\right) \Delta w+\frac{5 r^{2}+3 a^{2}}{r}(\Delta w)_{r}\right] \\
& -\frac{2 \sigma(\sigma-3)}{(\sigma+3)(\sigma-1)} a^{2} \Delta w+\frac{\sigma-1}{4(\sigma+3)} \frac{\left(r^{2}-a^{2}\right)^{2}}{r^{2}} \Delta w_{\theta \theta} .
\end{aligned}
$$

Proof: Let $u=r^{2} w_{r r}$ and $V=\Delta w$. Then conditions (3.16) and (3.17) may be written in the form

$$
\frac{1-\sigma}{a^{2}} r u_{r}+(\sigma-2) r V_{r}+(\sigma-1) V=0
$$




$$
\frac{1-\sigma}{a^{2}} u+\sigma V=0 \text { on } Q .
$$

Define the functions $m$ and $n$ by

$$
m=\frac{1-\sigma}{a^{2}} u+\sigma V-\frac{1-\sigma}{4 a^{2}}\left(r^{2}-a^{2}\right)\left[r V_{r}+2 V\right]
$$

and

$$
n=\frac{1-\sigma}{a^{2}} u+\frac{\sigma-3}{2} V-\frac{1-\sigma}{4 a^{2}}\left(r^{2}-a^{2}\right)\left[r V_{r}+2 V\right] .
$$

By direct computation, it is easily verified that these functions are harmonic in $D$. Now by (3.21) and Lemma 9, $m$ vanishes on $Q$ and hence, by Lemma 1, may be continued into $D^{*}$ by the relation

$$
m\left(p^{\prime}\right)=-m(p) .
$$

From the definition of $n$ we have, by (3.20) and Lemma 9,

$$
r n_{r}=\frac{1-\sigma}{a^{2}} r u_{r}+(\sigma-2) r V_{r}+(\sigma-1) V=0 \quad \text { on } \quad Q .
$$

By Lemma 2 such a function is continued into $D^{*}$ by the relation

$$
n\left(p^{\prime}\right)=n(p) \text {. }
$$

Having continued $m$ and $n$ into $D^{*}$, we shall now express $u$ in terms of $m$, $n, m_{r}$ and $n_{r}$ thus yielding a continuation of $u$. To do this let us use (3.22) and (3.23) to obtain the relation

$$
V=\frac{2}{\sigma+3}(m-n) .
$$

By differentiation of (3.27) we have

$$
r V_{r}=\frac{2}{\sigma+3}\left(r m_{r}-r n_{r}\right) .
$$

If we solve (3.22) for $u$ and use (3.27) and (3.28) we obtain the expression

$$
u=\frac{a}{1-\sigma}\left[\frac{3-\sigma}{\sigma+3} m+\frac{2 \sigma}{\sigma+3} n\right]+\frac{\left(r^{2}-a^{2}\right)}{2(\sigma+3)}\left(2 m+r m_{r}-2 n-r n_{r}\right) .
$$

Now, using (3.29), (3.26) and the relation $R \partial / \partial R=-r \partial / \partial r$, we have, as a continuation for $u$ in terms of $m$ and $n$,

$$
\begin{aligned}
u\left(p^{\prime}\right)=\frac{a^{2}}{1-\sigma}\left[\frac{\sigma-3}{\sigma+3} m+\right. & \left.\frac{2 \sigma}{\sigma+3} n\right] \\
& +\frac{a^{2}}{r^{2}} \frac{\left(r^{2}-a^{2}\right)}{2(\sigma+3)}\left(2 m+2 n-r m_{r}-r n_{r}\right) .
\end{aligned}
$$


Substituting (3.22) and (3.23) into (3.30), (3.19) results. Since $u=r^{2} w_{r r}$ is a biharmonic function which has been continued into $D^{*}$, it follows from the corollary of Lemma 5 that $w$ may be continued biharmonically into $D^{*}$. Thus $w$ may be considered to be defined as a biharmonic function throughout $D^{*}$.

To obtain (3.18) we consider the identity

$$
\int_{r}^{a} \rho w_{\rho \rho}(\rho, \theta) d \rho=a w_{r}(a, \theta)-r w_{r}-w(a, \theta)+w .
$$

This may be written as

$$
\frac{\partial}{\partial r}\left(r^{-1} w\right)=\frac{1}{r^{2}}\left(a w_{r}(a, \theta)-w(a, \theta)\right)-\frac{1}{r^{2}} \int_{r}^{a} \rho w_{\rho \rho}(\rho, \theta) d \rho .
$$

Integrating this expression from $r$ to $a$ yields

$$
w=r \int_{r}^{a} \rho^{-2}\left[\int_{\rho}^{a} \rho^{\prime} w_{\rho^{\prime} \rho^{\prime}}\left(\rho^{\prime}, \theta\right) d \rho^{\prime}\right] d \rho+(r-a) w_{r}(a, \theta)+w(a, \theta) .
$$

This may be integrated by parts and we obtain

$$
w=\int_{r}^{a} \rho w_{\rho \rho}(\rho, \theta) d \rho-r \int_{r}^{a} w_{\rho \rho}(\rho, \theta) d+(r-a) w_{r}(a, \theta)+w(a, \theta) .
$$

Substituting $u=r^{2} w_{r r}$ we have

$$
w=\int_{r}^{a} \frac{u}{\rho} d \rho-r \int_{r}^{a} \frac{u}{\rho^{2}} d \rho+(r-a) w_{r}(a, \theta)+w(a, \theta) .
$$

Since $u$ is defined at all points of $D^{*},(3.31)$ gives the continuation of $w$ into $D^{*}$. That is,

(3.32) $w\left(p^{\prime}\right)=\int_{R}^{a} \frac{u(\bar{R}, \theta)}{\bar{R}} d \bar{R}-R \int_{R}^{a} \frac{u(\bar{R}, \theta)}{\bar{R}^{2}} d \bar{R}+(R-a) w_{r}(a, \theta)+w(a, \theta)$, $w_{r}(a, \theta)$ and $w(a, \theta)$ being functions of $\theta$ only. Since $u(R, \theta)$ is expressed by (3.19) in terms of known functions defined in $D$, a change of variables in the integrals in (3.32) and use of the relation $r R=a^{2}$ will lead to (3.18). We have, changing variables of integration,

and

$$
\int_{R}^{a} \frac{u(\bar{R}, \theta)}{\bar{R}} d \bar{R}=-\int_{r}^{a} \frac{u\left(a^{2} / \rho, \theta\right)}{\rho} d \rho
$$

$$
R \int_{R}^{a} \frac{u(\bar{R}, \theta)}{\bar{R}^{2}} d \bar{R}=-\frac{1}{r} \int_{r}^{a} u\left(\frac{a^{2}}{\rho}, \theta\right) d \rho .
$$

Substitution of these expressions into (3.32) gives (3.18). Q.E.D.

\section{Sliding Clamped Edge.}

Theorem 4: Let $w$ be defined in $D$ and satisfy

$$
\Delta^{2} w=0 \text { in } D
$$




$$
\left.\begin{array}{rl}
(\Delta w)_{r}-(1-\sigma) \frac{1}{a^{3}} w_{\theta \theta} & =0 \\
w_{r} & =0
\end{array}\right\} \text { on } Q
$$

Then $w$ has a biharmonic continuation to $D^{*}$ given by

$$
\begin{aligned}
w\left(p^{\prime}\right)=w(p)-\frac{2}{1+\sigma} \int_{r}^{a} & {\left[(1-\sigma) w_{\rho}+a^{2} r^{-1} \Delta w\right] d \rho } \\
+ & \frac{1}{8}\left[\frac{3-\sigma}{1-\sigma}+\frac{a^{2}}{r^{2}}\right] r^{\frac{1}{2}(1+\sigma)} \int_{r}^{a}\left[4(1-\sigma) \rho w_{\rho}\right. \\
+ & \left.(\sigma-3)\left(\rho^{2}-a^{2}\right) \Delta w+4 \rho^{2} \Delta w\right] \rho^{-\frac{1}{2}(3+\sigma)} d \rho .
\end{aligned}
$$

Proof: By Lemma 6, $w$ has the representation

$$
w=h+\left(r^{2}-a^{2}\right) V
$$

in $D$, where $\Delta h=0$ and $\Delta V=0$ in $D$. Define $g$ as

$$
g=V+\frac{1-\sigma}{4 a^{2}} r h_{r} .
$$

Since $\Delta h=0, \Delta\left(r h_{r}\right)=0$ and hence $\Delta g=0$ in $D$. Substituting (3.37) into (3.36) we have

$$
w=h+\left(r^{2}-a^{2}\right)\left(g-\frac{1-\sigma}{4 a^{2}} r h_{r}\right) .
$$

Applying (3.33) to (3.38) we find that

$$
2 g_{r}+r g_{r r}+\frac{1}{a}\left(\frac{\sigma-1}{\sigma+1}\right) g_{\theta \theta}=0 \quad \text { on } \quad Q .
$$

This expression may be written in the form

$$
\frac{\partial}{\partial r}\left(\frac{1+\sigma}{2} g+r g_{r}\right)=0 \text { on } Q \text {. }
$$

Now by Lemma 2 , since $\Delta\left(\frac{1}{2}(1+\sigma) g+r g_{r}\right)=0$ in $D, \frac{1}{2}(1+\sigma) g+r g_{r}$ may be continued into $D^{*}$ by means of Lemma 2 .

From Lemma 4 it follows that $g$ may be continued into $D^{*}$.

From (3.34) and (3.38) it follows that

$$
(1+\sigma) r h_{r}+4 a^{2} g=0 \text { on } Q
$$

and hence, by Lemma $1,(1+\sigma) r h_{r}+4 a^{2} g$ may be continued into $D^{*}$. Again applying Lemma 4 we see that $h$ may be continued into $D^{*}$. Thus $w$ can be continued biharmonically into $D^{*}$ and is defined there by means of (3.38). For the remainder of the proof we may, as before, assume that $w$ satisfies the boundary conditions in the strict sense.

To express the continuation for $g$ we make use of (3.38), Lemma 2 and Lemma 
10 and write

$$
\frac{1+\sigma}{2} g\left(p^{\prime}\right)+R \frac{\partial g\left(p^{\prime}\right)}{\partial R}=\frac{1+\sigma}{2} g(p)+r \frac{\partial g(p)}{\partial r} .
$$

Making use of $R \partial / \partial R=-r \partial / \partial r,(3.40)$ may be solved for $g\left(p^{\prime}\right)$ yielding

$$
g\left(p^{\prime}\right)=g(p)+2 r^{\frac{1}{2}(1+\sigma)} \int_{r}^{a} \rho^{-\frac{1}{2}(1+\sigma)} g_{\rho}(\rho, \theta) d \rho .
$$

To continue $h$ we note that $(1+\sigma) r h_{r}+4 a^{2} g$ is harmonic in $D$ and vanishes on $Q$. Hence by Lemma 1 and Lemma 10 we may write

$$
(1+\sigma) R \frac{\partial h\left(p^{\prime}\right)}{\partial R}+4 a^{2} g\left(p^{\prime}\right)=-(1+\sigma) r \frac{\partial h(p)}{\partial r}-4 a^{2} g(p) .
$$

Using $R \partial / \partial R=-r \partial / \partial r$ we may write $(3.42)$ in the form

$$
\frac{\partial}{\partial r}\left(h\left(p^{\prime}\right)-h(p)\right)=\frac{4 a^{2}}{1+\sigma} \frac{1}{r}\left(g\left(p^{\prime}\right)+g(p)\right) .
$$

This expression may be integrated from $r$ to $a$ yielding

$$
h\left(p^{\prime}\right)=h(p)-\frac{4 a^{2}}{1+\sigma} \int_{r}^{a} \frac{1}{\rho}\left(g\left(p^{\prime}\right)+g(p)\right) d \rho
$$

since $h\left(p^{\prime}\right)=h(p)$ on $Q$.

Thus we have

$$
w\left(p^{\prime}\right)=h\left(p^{\prime}\right)+\left(R^{2}-a^{2}\right)\left(g\left(p^{\prime}\right)-\frac{1-\sigma}{4 a^{2}} R \frac{\partial h\left(p^{\prime}\right)}{\partial R}\right) .
$$

Combining (3.41), (3.44) and (3.45) we may write

$$
\begin{aligned}
w\left(p^{\prime}\right) & =h(p)-\frac{4 a^{2}}{1+\sigma} \int_{r}^{a} \frac{2 g(p)}{\rho} d \rho \\
& -\frac{4 a^{2}}{1+\sigma} \int_{r}^{a} 2 \rho^{\frac{1}{2}(\sigma-1)}\left[\int_{\rho}^{a} \rho_{1}^{-\frac{1}{2}(\sigma+1)} g_{\rho_{1}}(p) d \rho_{1}\right] d \rho \\
& -\frac{a^{2}}{r^{2}}\left(r^{2}-a^{2}\right) \frac{3-\sigma}{1+\sigma} g(p) \\
& -\frac{a^{2}}{r^{2}}\left(r^{2}-a^{2}\right)\left[\frac{4}{1+\sigma} r^{\frac{1}{2}(1+\sigma)} \int_{r}^{a} \rho^{-\frac{1}{2}(\sigma+1)} g(p) d \rho+\frac{1-\sigma}{4 a^{2}} r h_{r}(p)\right] .
\end{aligned}
$$

Expressions (2.13) and (2.14) with

$$
k=-\frac{1}{2 a^{2}}\left(\frac{1-\sigma}{2}\right)
$$

may now be inserted into (3.46) and after a lengthy but elementary computation (3.35) results. Q.E.D. 
Again it should be noted that no boundary terms occur in the final expression. Throughout this section it is assumed that $D$ is a simply connected region. We show now that the restriction is unnecessary.

Suppose $D^{\prime}$ is a region which satisfies all the requirements placed on $D$ but is not simply connected. (We may assume that $D^{\prime}$ is connected, otherwise each of the components of $D^{\prime}$ would be simply connected and could be treated individually.) It is clear then that if we remove the points having coordinates $(r, 0)$ from $D^{\prime}$, the resulting region $D^{\prime \prime}$ will be simply connected. Now let $w$ be biharmonic in $D^{\prime}$ and satisfy the boundary conditions of Theorem 1, 2, 3 or 4 . Then $w$ has a continuation formula from $D^{\prime \prime}$ to $D^{\prime \prime *}$. But $D^{\prime \prime}$ could have been defined by omitting points with coordinates of the form $(r, \pi)$ from $D^{\prime}$ (call the resulting region $\left.D^{\prime \prime \prime}\right)$, and the same formula gives the continuation into $D^{\prime \prime \prime *}$. Hence the formula is valid at all points of $D^{\prime}$ and the function $w$ can be continued by means of the appropriate formula into $D^{\prime *}$.

Theorem 1 can be proved in a way analogous to the proofs of Theorems 2 and 4. This method of proof is different from that given by DuFris [8].

In each of the cases discussed here expressions for the continuation of the derivatives of $w$ are easily written down. Hence, from these formulæ, the continuation of the moments and the stresses may be obtained.

\section{Remarks.}

(a) In Theorems 2, 3, and 4, $\sigma$ is assumed to be Poisson's ratio and hence is restricted to be between 0 and $\frac{1}{2}$. However, the theorems stated are mathematically valid for all other values of $\sigma$ with the following exceptions:

1. Theorem 2 is valid for all values of $\sigma$ but the proof presented is not correct for $\sigma=-1$. In this case one obtains the continuation for $w$ by applying (3.4) and (3.5) to (3.7) and using Lemmas 1 and 2. This leads to the simple formula

$$
w\left(p^{\prime}\right)=-\frac{a^{2}}{r^{2}} w(p)
$$

to which (3.6) reduces for $\sigma=-1$.

It should also be noted that, since this theorem holds for every value of $\sigma$, we could replace $\sigma$ by $\sigma+a c$, thus writing (3.4) as

$$
w_{r r}+\frac{\sigma}{r} w_{r}+c w_{r}=0 \text { on } Q .
$$

Physically then, this problem corresponds to the case in which $Q$ is elastically supported. When $c=0$ we again have the simply supported edge and when $c=\infty$ (or $\sigma=\infty$ ) (3.6) reduces to the formula for the clamped edge. This can easily be seen by expanding $w$ and $\Delta w$ in a power series about $r$ and integrating term by term. In each case the first term of the series is the only term which contributes as $\sigma \rightarrow \infty$.

2. Theorem 3 fails in case $\sigma=1$ or -3 . When $\sigma=1$, (3.16) and (3.17) 
become $(\Delta w)_{r}=0$ and $\Delta w=0$ on $Q$ respectively. Since $\Delta w$ is harmonic it follows from Lemma 3 that $\Delta w \equiv 0$ in $D$. But every harmonic function satisfies the hypothesis of Theorem 3 when $\sigma=1$. It is clear then that the continuation is not determined by these conditions alone.

The case $\sigma=-3$ is of some interest. Here the previously defined harmonic functions $m$ and $n$ are identical. The boundary conditions now state $m=m_{r}=0$ on $Q$. Again, from Lemma 3, we conclude that $m \equiv 0$ in $D$. Hence we see that $w$ must satisfy the third order equation

$$
r^{2} w_{r r}=\frac{1}{4}\left(r^{2}-a^{2}\right)\left[r(\Delta w)_{r}+2 \Delta w\right]+\frac{3}{4} a^{2} \Delta w \text { in } D .
$$

Clearly, any biharmonic solution of (3.47) satisfies (3.16) and (3.17) for $\sigma=-3$ and vice versa.

We shall now show that the conditions of the theorem, with $\sigma=-3$, are not sufficient to uniquely determine the continuation into $D^{*}$. Let $w$ be a biharmonic function in $D$, satisfying (3.16) and (3.17) $(\sigma=-3)$. By Lemma 6, $w$ has the representation

$$
w=V_{1}+\left(r^{2}-a^{2}\right) \frac{V_{2}}{a^{2}}
$$

in $D$, where $\Delta V_{1}=0$ and $\Delta V_{2}=0$ in $D$. By Lemma 4 (with $k=0$ ) there is a harmonic function $h$ such that

$$
r h_{r}=V_{1} \text { in } D
$$

Define the harmonic function

$$
g=V_{2}-h_{\theta \theta} \text {. }
$$

Then, putting (3.49) and (3.50) into (3.48), we have

$$
w=r h_{r}+\frac{r^{2}-a^{2}}{a^{2}}\left(g+h_{\theta \theta}\right) \text {. }
$$

Applying (3.16) and (3.17) to (3.51) we obtain

$$
\begin{aligned}
g_{r r} & =0, \\
g-r g_{r} & =0,
\end{aligned}
$$

respectively, on $Q$. (Note that $h$ does not appear in the boundary conditions.) But $g-r g_{r}$ is a harmonic function which vanishes together with its normal derivative on $Q$, since

$$
\frac{\partial}{\partial r}\left(g-r g_{r}\right)=-r g_{r r}
$$

Thus Lemma 3 applies and $g-r g_{r} \equiv 0$ throughout $D$. Since $g$ is a harmonic function $g$ has the form $g=A x+B y$ where $A$ and $B$ are constants. So any any biharmonic function satisfying (3.16) and (3.17) (for $\sigma=-3$ ) may be 
written

$$
w=r h_{r}+\frac{\left(r^{2}-a^{2}\right)}{a^{2}}\left(h_{\theta \theta}+A x+B y\right) .
$$

Thus, since the continuation of $w$ depends on the continuation of the harmonic function $h$, we conclude that there is no general formula which expresses the continuation of $w$.

A somewhat more general statement of Theorem 3 could have been given and still proved in the same manner. If we write for (3.20) and (3.21)

$$
c_{1} r u_{r}+c_{2} r V_{r}-a^{2} c_{1} V=0
$$

and

$$
c_{3} u+c_{4} V=0
$$

respectively, with $c_{1}, c_{2}, c_{3}$ and $c_{4}$ constants, and choose for $m$ and $n$

$$
\begin{aligned}
& m=c_{3} u+c_{4} V-\frac{c_{3}}{4}\left(r^{2}-a^{2}\right)\left[r V_{r}+2 V\right], \\
& n=c_{1} u+\frac{2 c_{2}+a^{2} c_{1}}{2} V-\frac{c_{1}}{4}\left(r^{2}-a^{2}\right)\left[r V_{r}+2 V\right]
\end{aligned}
$$

then a continuation can be obtained for $w$, provided $2 c_{2} c_{3}+a^{2} c_{1} c_{3}-2 c_{1} c_{4} \neq 0$. This can be done by exactly the same procedure as was used in the proof of Theorem 3. For $c_{1}=c_{3}=(1-\sigma) / a^{2}, c_{2}=\sigma-2$ and $c_{4}=\sigma$ we have again the free edge problem, and the stated condition yields

$$
2(\sigma-2) \frac{1-\sigma}{a^{2}}+\frac{(1-\sigma)^{2}}{a^{2}}-2 \frac{\sigma(1-\sigma)}{a^{2}} \neq 0
$$

which is the same as $(\sigma-1)(\sigma+3) \neq 0$.

3. Theorem 4 is valid for all values of $\sigma$; however, when $\sigma=-1$ the right hand side of (3.35) must be considered to be taken in the sense of the limit. The proof given is not valid for $\sigma=-1$ but the correct result may be obtained by writing

$$
w=h+\frac{\left(r^{a}-a^{2}\right)}{2 a^{2}} g
$$

and applying (3.33) and (3.34). From this we may deduce the continuations of $h$ and $g$. Thus, writing $w\left(p^{\prime}\right)$ in terms of $h(p)$ and $g(p)$ and substituting into this the expressions for the continuations of $h$ and $g$, we have

$$
\begin{aligned}
w\left(p^{\prime}\right)=w(p)+ & \left(\ln r^{2}-\frac{r^{2}-a^{2}}{r^{2}}\right) \int_{r}^{a}\left[w_{\rho}+\frac{a^{2}}{2 \rho} \Delta w\right] d \rho \\
& -2 \int_{r}^{a}\left[w_{\rho}+\frac{a^{2}}{2 \rho} \Delta w\right] \ln \rho d \rho+\frac{1}{2} \int_{r}^{a} \frac{\rho^{2}-a^{2}}{\rho} \Delta w d \rho .
\end{aligned}
$$


(b) In Theorems 2, 3, and 4 it is mathematically interesting to observe what happens if $\sigma$ is allowed to approach infinity.

1. Statement (3.4) of Theorem 2 reduces to $w_{r}=0$, since $w$ is assumed analytic in its domain of definition. Thus the hypothesis of Theorem 2 reduces to that of Theorem 1 and it is easy to check that (3.6) goes into (3.3).

2. In Theorem 3 the boundary conditions (3.16) and (3.17) can be stated as

$$
\frac{\partial^{2}}{\partial \theta^{2}}\left(w_{\theta \theta}+w\right)=0
$$

and

$$
\frac{\partial}{\partial r}\left(w_{\theta \theta}+w\right)=0 \quad \text { on } \quad Q
$$

(3.19) is easily evaluated as $\sigma \rightarrow \infty$ yielding the continuation for such a biharmonic function.

3. The boundary conditions in Theorem 4 , as $\sigma \rightarrow \infty$, become

$$
w_{\theta \theta}=0
$$

and

$$
w_{r}=0 \text { on } Q \text {. }
$$

The limit of the right hand side of (3.35) can be evaluated and is seen to be

$$
w\left(p^{\prime}\right)=-w(p)+2 w(a)+r^{-2}\left(r^{2}-a^{2}\right)\left[r w_{r}-\frac{1}{4}\left(r^{2}-a^{2}\right) \Delta w\right] .
$$

Formula (3.59) differs from (3.3) by the term $2 w(a)$ which is a function of $\theta$ alone. In case $w=0$ on $Q$ this term disappears and (3.59) and (3.3) are identical.

(c) The problems treated here can be reduced to those of the half plane by the following procedure. Choose the origin at the point $x=a, y=0$ thus having $r^{2}=(x-a)^{2}+y^{2}$, and then allow $a$ to approach infinity. The circle and its interior, $r \leqq a$, will be transformed into the right half plane $x \geqq 0$. By such a process, the results obtained reduce to those reached by Dufris [8] in the analogous half plane problems.

\section{Conclusion}

Aside from the fact that reflection principles are of interest from the point of view of analytic continuation, it is interesting to note a few applications which have been made. HuBER in [17] uses a reflection principle for solutions of equation (1.3) in order to prove a uniqueness theorem for these solutions. An example of the use of reflection in the determination of the solution of a boundary value problem may be found in a paper by the author [3]. Still another example is given by PoRITsky [28] where he uses the reflection principle for $\Delta^{2} u=0$, under the conditions $u=\partial u / \partial r=0$ on a circle, to construct a "Green's function" for 
the region exterior to the circle. It is rather interesting that these examples differ considerably from each other.

In this paper we have not attempted to give the most general region $D$ for which the reflection principles are valid. For example, in some cases the origin could be included in the region. The region was chosen to be of this type for convenience and simplicity.

It should be noted that all results remain valid if $D$ is chosen to be the intersection of $D^{*}$ with $r>a$ and required to be finite.

\section{Bibliography}

[1] Armansi, E., Sull'integrazione dell'equazione differenziale $\Delta^{2 n}=0$, Annali di matematica, serie III, 2, 1899, pp. 1-59.

[2] Boggio, T., Sulla deformazione delle piastre elastiche soggette al calore, Atti R. Acc. Scienze Torino, 40, 1904-1905.

[3] Bramble, J., The thick elastic spherical shell under concentrated torques, Technical Note BN-54, University of Maryland, 1955.

[4] Coluccr, A., Teoremi e problemi sulle funzioni iperamoniche trattati col metodo degli operator lineari, Rend. Acc. Sc. Fis. Mat. Napoli, 7, 1937, pp. 48-54.

[5] CoLuccr, A., Sulla rappresentazione delle funzione iperamoniche a mezzo di armoniche, Rend. Acc. Sc. Fis. Mat. Napoli, 7, 1937, pp. 55-62.

[6] Courant, R. \& Hilbert, D., Methoden der Mathematischen Physik, Springer, Berlin, 1937.

[7] Diaz, J. B. \& Ludford, G. S. S., Reflection principles for linear elliptic second order partial differential equations with constant coefficients, Annali di Matematica Pura ed Applicata, Serie IV, 39, 1955, pp. 87-95.

[8] DuFris, R. J., Continuation of biharmonic functions by reflection, Duke Math. Journal, 22, 1955, pp. 313-324.

[9] Duffin, R. J., Analytic continuation in elasticity, Journal of Rational Mechanics and Analysis, 5, 1956, pp. 939-950.

[10] Fichera, G., Sviluppi in serie e teoremi di decomposizione in somme per le funzioni iperharmoniche, Rend. Circ. Mat. Palermo, 63, 1941, pp. 41-64.

[11] Fichera, G., Decomposizione al modo di Poincaré delle funzione bi-iperamoniche in due variabili, Rend. Acc. Sc. Fis. Mat. Napoli, 11, 1940-41, pp. 134-149.

[12] Fichera, G., Un teorema generale sulla struttura delle funzione iperamoniche, Rend. Acc. d'Italia, 3, 1942, pp. 511-523.

[13] Garabedian, P. R., Applications of analytic continuation to the solution of boundaryvalue problems, Journal of Rational Mechanics and Analysis, 3, 1954, pp. 383-393.

[14] HiLbert, D., Grundzüge einer allgemeinen Theorie der linearen Integralgleichungen, Nachrichten der K. Gesellschaft der Wissenschaften zu Göttingen, Mathematische-physikalische Klasse, 1910.

[15] Hohersel, G., Jahresbericht der deutschen Mathematiker-Vereinigung, 39, 1930, p. 54.

[16] HuBer, A., On the reflection principle for polyharmonic functions, Communications on Pure \& Applied Math., 9, 1956, pp. 471-478.

[17] HUBER, A., On the uniqueness of generalized axially symmetric potentials, Annals of Math., 60, 1954, pp. 351-358.

[18] Jонn, F., Prolongement et reflexion des solutions des équations aux dérivées partielles, Colloques Internationaux du Centre National de la Recherche Scientifique, Nancy, 1956, pp. 103-109.

[19] KellogG, O. D., Foundations of Potential Theory, Springer, Berlin, 1929. 
[20] LEwy, H., A theory of terminals and the reflection laws of partial differential equations, Technical Report No. 4, Stanford Univ., 1952.

[21] Love, A., The Mathematical Theory of Elasticity, Dover, New York, 1944.

[22] Nicolesco, M., Nouvelles recherches sur les functions polyharmoniques. Disquisitions Math. Phys., 1, 1940, pp. 43-74.

[23] Nicolesco, M., Les fonctions polyharmoniques, Actualités scientifiques et industrielles, 331, Hermann, Paris, 1936.

[24] Nicolesco, M., Sur l'unicité de la representation des fonctions harmoniques d'orde $p$ au moyen des fonctions harmonique d'ordre un, Bull. Math. Soc. Roumaine Sc., 37, 1935, pp. 83-87.

[25] Prcone, M., Nuovi indirizzi di ricerca nella teoria e nel calcolo delle soluzioni di taluneequazioni lineari alle derivate parziali della Fisica matematica, Ann. Sc. N. Sup. Pisa, 5, 1936, pp. 213-288.

[26] Ponitsky, H., On Reflection of Singularities of Harmonic Functions corresponding to the Boundary Condition $\partial u / \partial n+a u=0$, A.M.S., Dec., 1937, pp. 873-884.

[27] Poritsky, H., On the Boundary Condition $\partial u / \partial n+a u=0$ for Harmonic Functions, A.M.S., June, 1938, pp. 443-448.

[28] Poritsky, H., Application of analytic functions to two dimensional biharmonic analysis, Trans. A.M.S., 59, 1946, pp. 248-279.

[29] SchröDer, K., Zur Theorie der Randwertaufgaben der Differentialgleichung $\Delta \Delta u=0$, Math. Zeit., 48, 1943, pp. 553-675.

[30] Schwarz, H. A., Ueber die Integration der Differential gleichung $\partial^{2} u / \partial x^{2}+\partial^{2} u / \partial y^{2}=0$ unter vorgeschreibenen Grenzung Unstetigkeitsbedingungen, Monatsberichte der Königlichen Akademie der Wissenschaften zu Berlin, 1870, pp. 767-795.

[31] Sokolnikoff, I. S., Mathematical Theory of Elasticity, McGraw-Hill, New York, 1956.

[32] Tоцотті, C., Sulla struttura della funzioni iperarmoniche in piú variabili independenti, Giorn. Mat. Battaglini, 77, 1947-48, pp. 61-117.

[33] Wernstock, R., Calculus of Variations, McGraw-Hill, New York, 1952.

\author{
University of Maryland \\ College Park, Maryland \\ and \\ General Electric Company \\ Cincinnati, Ohio
}

